\title{
Corrigendum
}

\section{Beyond immune density: critical role of spatial heterogeneity in estrogen receptor-negative breast cancer}

Sidra Nawaz, Andreas Heindl, Konrad Koelble and Yinyin Yuan

Modern Pathology (2015) 28, 1621; doi:10.1038/modpathol.2015.133

Correction to: Modern Pathology (2015) 28, 766-777; doi:10.1038/modpathol.2015.37; published online 27 February 2015

In this paper, the 'Getis-Ord Spatial Statistics for Hotspot Identification' subsection on page 768 , the first equation should be

$z_{i}=\frac{\sum_{j=1}^{n} w_{i, j} c_{j}-\bar{c} \sum_{j=1}^{n} w_{i, j}}{S U}$

The supplementary file has been updated to concur with the online guide on the authors' website for reproducing these results. The changes are concerned with the $\mathrm{R}$ code used to generate plots: (i) additional lines have been added to the code to calculate $P$-values and patient numbers and deaths shown in the figures, (ii) the code for plotting Figure 6 has been modified to produce the subplots in the same order as in the paper, and (iii) new format of the data matrix clearly defining which patients belong to the discovery or validation cohort. 